\title{
Influence of the Recirculation Zones on the Mass Transfer for a Drug- Eluting Stent
}

\author{
F. Chabi ${ }^{1}$, S. Champmartin ${ }^{1}$, C. Sarraf ${ }^{2}$ \\ ${ }^{1}$ ENSAM Angers \\ 2 bd du Ronceray, 49035 Angers, France \\ fatiha.chabi@ensam.eu; stephane.champmartin@ensam.eu \\ ${ }^{2}$ ENSAM Paris \\ 151 bd de l'hôpital, 75013 Paris, France \\ christophe.sarraf@ensam.eu
}

\begin{abstract}
The disturbance of the blood flow close to the struts of a drug-eluting stent plays a leading role on the drug release towards the lumen and the arterial wall. The intra-stent flow is characterized by two recirculation zones upstream and downstream of the strut. The size of these stagnant regions depends on the flow parameters (Reynolds and Womersley numbers) and on geometrical parameters (stent design, artery diameter...). For the steady flow in an artery when the Reynolds number increases, the size of the downstream recirculation zone in the flow direction linearly increases and the size of the upstream recirculation zone decreases. The mass transfer in the vicinity of the stent strut is strongly coupled with the flow in these recirculation zones. Indeed these regions are places where high concentrations of the active ingredient can occur. They control the mean concentration and the spatial distribution of the drug in the arterial wall close to the stent. The upstream recirculation zone plays the role of a drug source diffusing the active ingredient towards the arterial wall. Inversely the downstream recirculation zone acts like a drug sink removing the active ingredient from the arterial wall. Far from the stent, the drug concentration in the arterial wall is mainly controlled by the mass transfer coming from the top of the stent.
\end{abstract}

Keywords: intra-stent flow, mass transfer, recirculation zones, active ingredient

\section{Introduction}

Drug eluting stents (DES) are commonly used in the treatment of coronary diseases and particularly to prevent intrastent restenosis. Today more than 500000 DES are implanted in the USA every year [1]. They are made of a metal scaffold coated with a polymer film acting like a reservoir of a pharmacologic agent. The efficiency of such a system is based on the transport of the active agent from the polymer towards the arterial tissue for an extended period of time. Several parameters are involved in this process: the nature of the drug and of the polymer, the stent design and the blood flow in this area. In vitro [2- 4] and in vivo studies [5-7] have highlighted the role of the coating, the drug, its concentration, the drug release kinetics in transporting the drug towards the arterial wall. Nevertheless these studies often give only qualitative information and are for some of them not directly usable for the optimization of DES. Beside these studies, the numerical simulations are more and more helpful to provide answers in the understanding of such complex problems. Among the many numerical studies available, the influence of the intra-stent flow (recirculation zones, pulsatility...) is rarely studied [8]. In this paper, we investigate the influence of the Reynolds number on the recirculation zones and on their coupling with the mass transfer in a left coronary artery.

\section{Numerical Models}

In this study, we model the flow and the mass transfer corresponding to a human left coronary artery. We use a 2D axisymmetric geometric model of an artery equipped with a drug eluting stent (Fig.1). The system consists of the arterial lumen, the arterial wall and a single drug eluting stent strut. This latter has a square cross-section of side $a=100 \mu \mathrm{m}$ in contact with the vascular wall. The arterial wall is supposed to be homogeneous, impermeable and with thickness $e=$ $5 \mathrm{~mm}$. The faces of the drug eluting stent strut noted "a", "p" and "d" are in direct contact with the blood flow and that in 
direct contact with the arterial wall is denoted "w". The "Ansys Fluent" software based on the finite volume method is used to simulate mass transfer coupled with the flow.

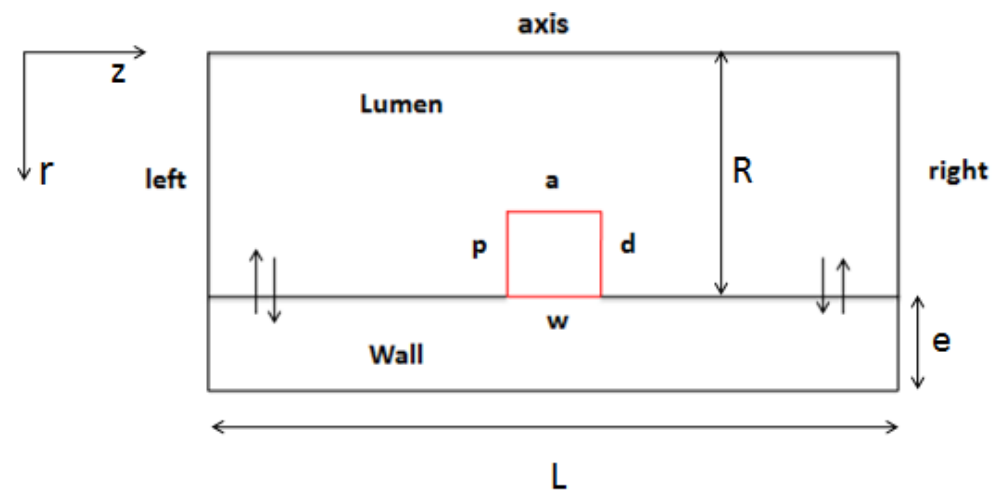

Fig. 1: Fluid domain (lumen) and solid (arterial wall) and stent.

The flow in the arterial lumen is assumed to be laminar, steady, incompressible and axisymmetric. The blood is assumed to be Newtonian. The boundary condition for the velocity profile at the inlet and outlet of the lumen domain is the Poiseuille profile:

$$
u_{z}(r)=2 V_{0}\left(1-\frac{r^{2}}{R^{2}}\right)
$$

With $V_{0}=0.16 \mathrm{~m} / \mathrm{s}$ the mean velocity of flow in a left coronary artery [9]. The steady mass transfer in the arterial lumen is governed by the advection-diffusion equation:

$$
u_{r} \frac{\partial C}{\partial r}+u_{z} \frac{\partial C}{\partial z}=D_{f}\left(\frac{1}{r} \frac{\partial}{\partial r}\left(r \frac{\partial C}{\partial r}\right)+\frac{\partial^{2} C}{\partial z^{2}}\right)
$$

With $D_{f}$ the diffusion coefficient in the arterial lumen, $C$ is the local concentration of active ingredient and $u_{z}, u_{r}$ are the axial and radial components of the velocity vector $\vec{u}$. In the arterial wall, the steady mass transfer is governed by the diffusion equation:

$$
\frac{1}{r} \frac{\partial}{\partial r}\left(r \frac{\partial C}{\partial r}\right)+\frac{\partial^{2} C}{\partial z^{2}}=0
$$

For the boundary conditions on the stent (faces "p", "d", "a" and "w") the no-slip condition for velocity and the normalized concentration $(\mathrm{C}=1)$ are imposed. At the inlet and outlet of the domain (faces "left" and "right") a zero concentration $(\mathrm{C}=0)$ for the mass transfer is applied. On the proximal and distal wall-lumen interfaces $(\mathrm{r}=\mathrm{R})$, the boundary condition type "interface" is applied. This condition allows the continuity of the mass flow between the two "fluid" and "solid" domains. On the outer surface of the arterial wall in the solid domain $(r=R+e)$, the adiabaticity condition is imposed (zero mass flux) and on the side surfaces of the solid domain $(\mathrm{z}=0$ and $\mathrm{z}=\mathrm{L})$, a zero concentration is imposed.

\section{Results and Analysis}

Figure 2 on the left shows the concentration field in the lumen and in the arterial wall as well as the streamlines for $R e=106$ in the immediate vicinity of the stent. We note that there is a coupling between the proximal and distal recirculation cells and the concentration of the active ingredient in the arterial wall. In the immediate vicinity of the stent, these recirculation flows disrupt the distribution of the concentration in the arterial wall under the stent. The proximal cell is small in size but is characterized by an area of high concentration "quite extensive". The distal cell is larger in size but 
characterized by a zone of high concentration "less extensive" than upstream of the stent. This results, under the stent at the interface "stent-arterial wall ", in an asymmetry of the concentration field slightly deformed towards the proximal side.
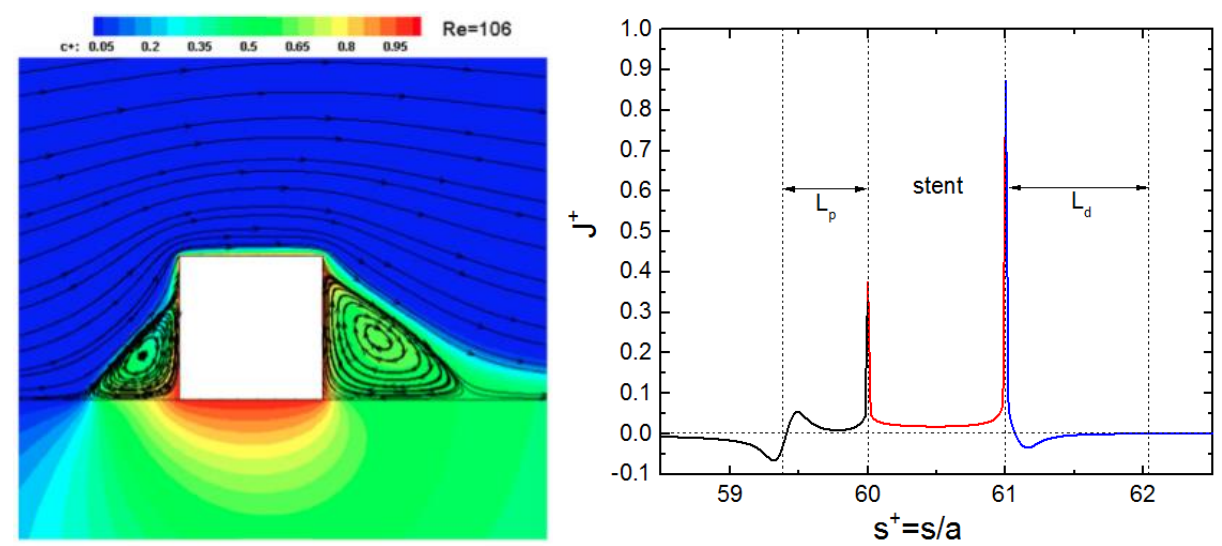

Fig. 2: Iso-concentration values and recirculation zones and mass flux density on the arterial wall at $\mathrm{Re}=106$.

Recirculation cells act in a different way for mass transfer. Figure 2 on the right shows the normalized mass flux density $\mathrm{J}^{+}$along the interface "lumen-wall" for $\mathrm{Re}=106$. In this figure, $L_{p}$ and $L_{d}$ represent respectively the lengths of the recirculation cells on the proximal and distal side. We see that $\mathrm{J}^{+}>0$ in the proximal recirculation cell which corresponds to a flux directed from the arterial lumen to the arterial wall. Therefore this zone acts as a localized source of active ingredient for the arterial wall. Conversely in the distal recirculation cell, over a large part of the lumen-arterial wall interface, $\mathrm{J}^{+}<0$ which corresponds to a mass flux directed from the arterial wall to the lumen. This zone therefore acts as a localized sink of active ingredient for the arterial wall. In Figure 3 we display the iso-values of concentration for Re $=$ 106 and $\mathrm{Re}=265$. The concentration in the recirculation zones and in the arterial wall decreases as Re increases. Far from the stent, the concentration field is not symmetrical with a higher concentration on the distal side. This asymmetry results in the coupling between flow and mass transfer. As we have shown in another study [10], the convection stretches the distal cell and contracts the proximal cell. The mass transfer coupled to these flows therefore follows the same behavior. In addition, the concentration field downstream of the stent strut is mainly due to the mass transfer from the face "a", which creates an area of higher concentration at the arterial wall which accentuates the asymmetry of the concentration field, on the distal side.

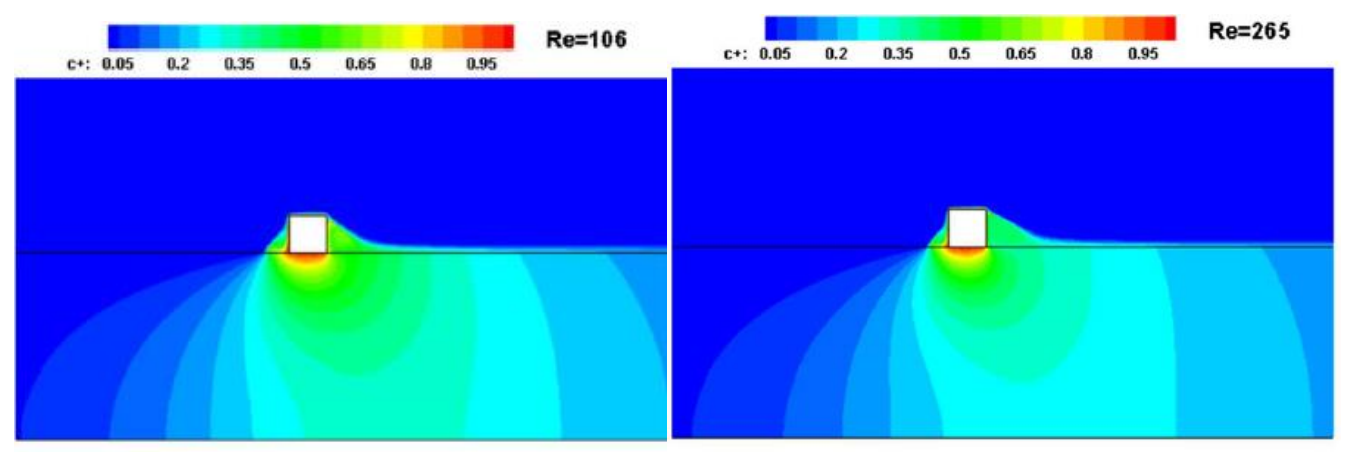

Fig. 3: Iso-concentration values for Reynolds numbers $\mathrm{Re}=106$ and $\mathrm{Re}=265$.

To quantify this phenomenon, we have plotted in Figure 4 the mean concentration $\bar{C}_{t}$ in the arterial wall as a function of the Reynolds number:

$$
\bar{C}_{t}=\frac{1}{V_{t}} \iiint C(r, z) d V
$$


With $V_{t}$ the volume of the arterial wall. A detailed analysis shows that the average concentration $\bar{C}_{t}$ is essentially due to the concentration field downstream of the stent and particularly to that located downstream of the distal recirculation cell which results in the transfer of mass coming from face "a" of the stent. The overall decrease in $\overline{C_{t}}$ when Re increases is related to the increase of the mass flux and to that of the convective mixture.

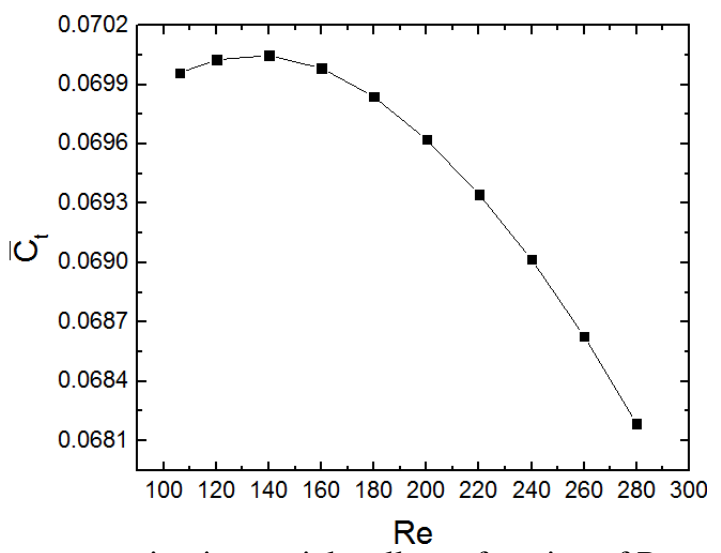

Fig. 4: Mean concentration in arterial wall as a function of Reynolds number.

\section{Conclusion}

In this paper we studied the coupling between the recirculation cells upstream and downstream of a stent strut and the spatial distribution of the active ingredient in the lumen and in the arterial wall. When Re increases, the size of the proximal cell decreases while that of the distal cell increases, which has an impact on the distribution of the concentration in the lumen and the arterial wall. In the immediate vicinity of the stent, these cells disrupt the mass transfer of the active ingredient to the arterial wall. The proximal cell acts as a source of active ingredient with a transfer directed from the lumen to the arterial wall. The distal cell acts as a sink of active ingredient with a transfer directed from the arterial wall to the lumen. Beyond the recirculation cells in the distal region, the concentration field is mainly governed by the mass flux coming from face "a" of the stent which is in direct contact with the main flow.

\section{References}

[1] G. G. Stefanini and D. R. Holmes Jr, "Drug-eluting coronary-artery stents," J. N Engl Med., vol. 368, no. 3, pp. 254265, 2013.

[2] S. V. Ranade, K. M. Miller, R. E. Richard, A. K. Chan, M. J. Allen, and M. N. Helmus, "Physical characterization of controlled release of paclitaxel from the taxus ${ }^{\mathrm{TM}}$ express $^{2 \mathrm{TM}}$ drug-eluting stent," J. Biomed Mater Res A., vol. 71, no. 4, pp. 624-634, 2004.

[3] S. Venkatraman and F. Boey, "Release profiles in drug-eluting stents: issues and uncertainties," J. Control Release., vol. 120, no. 3, pp. 149-160, 2007.

[4] R. Wessely, A. Schömig, and A. Kastrati, "Sirolimus and paclitaxel on polymer-based drug-eluting stents: similar but different," J. Am Coll Cardiol., vol. 47, no. 4, pp. 708-714, 2006.

[5] M. A. Lovich and E. R. Edelman, "Mechanisms of transmural heparin transport in the rat abdominal aorta after local vascular delivery," J. Circ Res., vol. 77, no. 6, pp. 1143-1150, 1995.

[6] C. W. Hwang, A. D. Levin, M. Jonas, H. Li, and E. R. Edelman, "Thrombosis modulates arterial drug distribution for drug-eluting stents," J. Circulation., vol. 111, no. 13, pp. 1619-1626, 2005.

[7] A. R. Tzafriri, N. Vukmirovic, V. B. Kolachalama, I. Astafieva, and E. R. Edelman, "Lesion complexity determines arterial drug distribution after local drug delivery," J. Control Release., vol. 142, no. 3, pp. 332-338, 2010.

[8] V. B. Kolachalama, A. R. Tzafriri, D. Y. Arifin, and E. R. Edelman, "Luminal flow patterns dictate arterial drug deposition in stent-based delivery," J. Control Release., vol. 133, no. 1, pp. 24-30, 2009.

[9] N. Bénard, “Analyse de l'écoulement physiologique dans un stent coronarien," Ph.D. dissertation, Univ. Poitiers.

[10] F. Chabi, S. Champmartin, C. Sarraf, and R. Noguera, "Critical evaluation of three hemodynamic models for the numerical simulation of intra-stent flows," J. Biomech., vol. 48, no. 10, pp. 1769-1776, 2015. 